\title{
Initial periodontal treatment affects nucleotide-binding domain leucine-rich repeat-containing protein 3 inflammasome priming in peripheral blood mononuclear cells
}

\author{
Kanako Higuchi, Ziauddin SM, Yasunori Yamashita, Yukio Ozaki, Atsutoshi Yoshimura* \\ Department of Periodontology and Endodontology, Graduate School of Biomedical Sciences, Nagasaki University, Nagasaki, Japan
}

\section{A R T I C L E I N F O}

\section{Keywords:}

Chronic periodontitis

Inflammation

Inflammasome

Interleukin-1 $\beta$

Mononuclear leukocyte

\begin{abstract}
A B S T R A C T
Objective: Accumulating evidence suggests an association between periodontitis and several systemic diseases, such as atherosclerosis. In the lesions of these diseases, nucleotide-binding domain leucine-rich repeat-containing protein 3 (NLRP3), apoptosis-associated speck-like protein containing a caspase activation and recruitment domain (ASC) and caspase-1 form inflammasome complex, which leads to the functional maturation of interleukin (IL)-1 $\beta$ via cleavage of caspase- 1 in macrophages. IL-1 $\beta$ plays a critical role in the etiology of these diseases; however, inflammasome priming - specifically, $L L-1 \beta$ and NLRP3 upregulation-is necessary for effective IL-1 $\beta$ production. We investigated the effect of initial periodontal treatment on the inflammasome priming of peripheral blood mononuclear cells (PBMCs).

Methods: Twenty-two patients with chronic periodontitis were enrolled in this study and given initial periodontal treatment. Peripheral blood samples were collected at baseline and re-evaluation $(41.1 \pm 29.1 \mathrm{~d}$ after the treatment), and the relative expression of $I L-1 \beta$, and three inflammasome components, ASC, NLRP3 and Caspase1, mRNA was determined using quantitative reverse transcription PCR. PBMCs were stimulated with silica crystals, and the IL-1 $\beta$ secretion was measured via enzyme-linked immunosorbent assay.

Results: Probing pocket depth and bleeding on probing (BOP) were significantly improved after the treatment. Expression of $I L-1 \beta$ and $A S C$ in the PBMCs decreased after the treatment. PBMCs stimulated with silica crystals secreted IL-1 $\beta$. The treatment attenuated IL-1 $\beta$ secretion by PBMCs in low BOP percentages group whereas IL- $1 \beta$ secretion was increased in high BOP percentages group.

Conclusion: Periodontal treatment altered the inflammasome priming status of the PBMCs, however, the effects on systemic diseases need to be further investigated.
\end{abstract}

\section{Introduction}

Periodontitis is a chronic inflammatory disease that causes destruction of periodontal tissue. Bacteria in the dental plaque trigger host inflammatory responses and induce the recruitment of leukocytes into the periodontal tissue. The infiltrated leukocytes release inflammatory cytokines that promote inflammation in the periodontal tissue. Growing evidence suggests that periodontitis is associated with several systemic inflammatory diseases, such as atherosclerosis, type 2 diabetes mellitus, rheumatoid arthritis, preterm birth, and Alzheimer's disease (Falcao \&
Bullón, 2019; Gaur \& Agnihotri, 2015). A meta-analysis found that periodontal treatment improves endothelial function and leads to a reduction in the biomarkers of atherosclerotic disease (Teeuw et al., 2014). An experimental mouse model showed that periodontitis exacerbated aspects of Alzheimer's disease (Ishida et al., 2017). These findings suggest the causal role of periodontitis in these systemic diseases. A common characteristic of periodontitis-associated systemic diseases is a noninfectious inflammation in the disease lesions. This sterile inflammation is triggered by cholesterol crystals, islet amyloid polypeptide, ATP, hypoxia, reactive oxygen species, uric acid, saturated

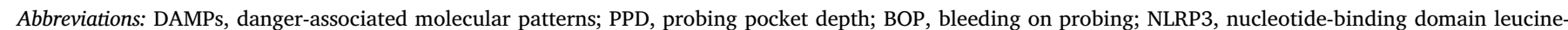

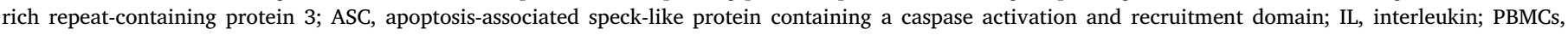

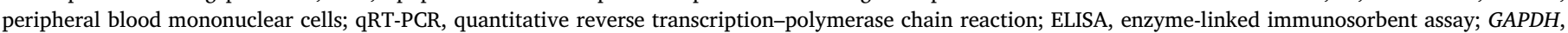
glyceraldehyde-3-phosphate dehydrogenase

* Corresponding author at: Department of Periodontology and Endodontology, Nagasaki University Graduate School of Biomedical Sciences, 1-7-1 Sakamoto, Nagasaki 852-8588, Japan.

E-mail address: ayoshi@nagasaki-u.ac.jp (A. Yoshimura). 
fatty acids, glucose, or amyloid $\beta$ (Patel et al., 2017). These molecules are called danger-associated molecular patterns (DAMPs). DAMPs activate the nucleotide-binding domain leucine-rich repeat-containing protein 3 (NLRP3) in the cytosol, and they promote the recruitment of apoptosis-associated speck-like protein containing a caspase activation and recruitment domain (ASC) and caspase-1 into a multiprotein complex known as the inflammasome (He, Hara, \& Núñez, 2016). Assembly of the NLRP3 inflammasome promotes self-cleavage of caspase1 , which in turn cleaves pro-interleukin (IL)- $1 \beta$ to facilitate its secretion and biological activity. Inflammasome-derived mature IL-1 $\beta$ is a potent proinflammatory mediator and has been shown to promote sterile inflammatory disease pathogenesis at multiple levels (Lukens, Gross, \& Kanneganti, 2012). For example, IL-1 $\beta$ can directly cause tissue destruction, altered fibroblast proliferation, and collagen deposition. Engagement of the IL-1 $\beta$ and IL-1 receptors promotes the production of secondary proinflammatory molecules, such as tumor necrosis factor- $\alpha$ and IL-6, which can trigger additional immune cell recruitment and activation. Stroke patients treated with an IL-1 receptor antagonist exhibited reduced levels of proinflammatory cytokines and improved cognitive functions (Emsley et al., 2005). Low-density lipoprotein receptor-deficient mice (genetically prone to atherosclerosis) that are reconstituted using bone marrow cells from mice lacking NLRP3, ASC, or $I L-1 \beta$ are remarkably resistant to atherosclerotic plaque formation (Duewell et al., 2010). Combined, this evidence indicates that NLRP3 inflammasome formation and IL-1 $\beta$ production play critical roles in the pathogenesis of these systemic diseases.

Before a functional NLRP3 inflammasome can be formed, transcriptional and posttranslational mechanisms must achieve a reasonable level of NLRP3 expression within the cell, and a suitable level of $I L$ $1 \beta$ must also be achieved (Patel et al., 2017). This process is called inflammasome priming. Expression of $I L-1 \beta$ and $N L R P 3$ can be induced by priming with microbial components, such as Toll-like receptor ligands, or endogenous molecules, such as tumor necrosis factor- $\alpha$ or IL$1 \beta$, through the activation of NF-kB (He et al., 2016). The oral cavity, and in particular periodontal pockets, could act as a natural reservoir of microorganisms (Falcao \& Bullón, 2019). These oral bacteria and their products, such as endotoxins, may enter the circulation and prime the inflammasome. Alternatively, the inflammatory cytokines or inflammatory mediators induced in diseased periodontium may have the potential to prime the inflammasome. Therefore, we hypothesized that inflammation in the periodontal tissue primes the inflammasome in peripheral blood mononuclear cells (PBMCs) and contributes to the exacerbation of systemic diseases. The aim of this study was to investigate the effect of initial periodontal treatment on inflammasome priming in PBMCs.

\section{Materials and methods}

\subsection{Sample collection}

Twenty-five patients with periodontitis who visited Nagasaki University Hospital between 2016 and 2017 were enrolled in this study, although three dropped out during the initial periodontal treatment, leaving 22 ( 4 males and 18 females, mean age: $60.5 \pm 15.2$ y) whose samples were analyzed. The inclusion criteria were: 1) possession of $\geq 15$ teeth; 2) an age of $\geq 20 \mathrm{y}$; and 3) $\geq 2$ teeth with attachment loss and alveolar bone resorption. The exclusion criteria were: 1 ) pregnancy or lactation; 2) renal or liver disease; 3) treatment with antibiotics within the previous month; 4) periodontal treatment within the previous three months; and 5) participation in another clinical study within the previous four months. Three periodontally and systemically healthy volunteers ( 2 males and 1 female, mean age: $29.6 \pm 1.5$ y) were recruited to obtain the control saliva and blood samples. Written informed consent was obtained from all participants. The design of the study and procedures for obtaining informed consent were approved by the Institutional Review Board of Nagasaki University. The study conforms to STROBE guidelines.

As part of a routine periodontal examination, probing pocket depth (PPD) and bleeding on probing (BOP) were measured at six aspects of each tooth before any periodontal treatment was performed and after the initial periodontal treatment. One $\mathrm{mL}$ of saliva was collected from each participant via passive secretion into a sterile tube. The saliva samples were stored at $-80{ }^{\circ} \mathrm{C}$ until use.

Human PBMCs were isolated from the venous blood before and after periodontal treatment. The PBMCs were isolated from a density gradient prepared using Ficoll-Paque Plus (GE Healthcare Bio-Science AB, Pittsburg, PA), and resuspended in RPMI 1640 medium supplemented with $10 \%$ FBS, $100 \mathrm{U} / \mathrm{mL}$ penicillin, and $100 \mu \mathrm{g} / \mathrm{mL}$ streptomycin.

\subsection{Quantitative reverse transcription-polymerase chain reaction ( $q R T$ - PCR) analysis}

To analyze $I L-1 \beta, N L R P 3, A S C$, and Caspase-1 mRNA levels, total RNA was extracted from the PBMCs using the RNeasy Mini Kit (Qiagen, Hilden, Germany) with on-column DNase treatment according to the manufacturer's instructions. For each sample, $24 \mathrm{ng}$ of total RNA was converted to first-strand cDNA using avian myeloblastosis virus reverse transcriptase (Promega, Madison, WI) at $25{ }^{\circ} \mathrm{C}$ for $10 \mathrm{~min}$, followed by $50 \mathrm{~min}$ at $42{ }^{\circ} \mathrm{C}$, and then $15 \mathrm{~min}$ at $70{ }^{\circ} \mathrm{C}$, using a Takara PCR thermal cycler (Takara Bio, Otsu, Japan). The cDNA was treated with RNase $\mathrm{H}$ and purified using the QIAprep Spin Miniprep Kit (Qiagen). The primer sequences used were as follows: $I L-1 \beta$ forward, $5^{\prime}$-GCTGGAGGACTTT AAGGGTTAC- $3^{\prime}$, and reverse, 5'-GATGTCTGGGTCTTGGTTCTC-3'; NLRP3 forward, 5'-AGCACCAGCCAGAGTCTAAC-3', and reverse, 5'-CCCCAACCACAATCTCCGAAT-3'; ASC forward, 5'-AACCCAAGCAA GATGCGGAAG-3', and reverse, 5'-TTAGGGCCTGGAGGAGCAAG-3'; Caspase-1 forward, 5'-CAAGGGTGCTGAACAAGG-3', and reverse, 5'-GGGCATAGCTGGGTTGTC-3'; glyceraldehyde-3-phosphate dehydrogenase $(G A P D H)$ forward, 5'-CATGTTCGTCATGGGTGTGA-3, and reverse, 5'-CAGGGGTGCTAAGCAGTTGG-3' (Feria, Taborda, Hernandez, \& Rugeles, 2018; Tzach-Nahman et al., 2017). Comparative quantification by PCR was performed with SYBR Premix Ex Taq using the Mx3000 P qPCR System (Agilent technologies, Santa Clara, CA). The amplification conditions were as follows: $95{ }^{\circ} \mathrm{C}$ for $10 \mathrm{~s}$, followed by 40 cycles of $95{ }^{\circ} \mathrm{C}$ for $5 \mathrm{~s}$ and $60^{\circ} \mathrm{C}$ for $20 \mathrm{~s}$, followed by a final cycle of $95{ }^{\circ} \mathrm{C}$ for $1 \mathrm{~min}, 55{ }^{\circ} \mathrm{C}$ for $30 \mathrm{~s}$, and $95^{\circ} \mathrm{C}$ for $30 \mathrm{~s}$. A melting curve analysis was used to confirm that the proper PCR products were amplified in all samples. The relative expression ratios of $I L-1 \beta, N L R P 3$, ASC, and Caspase-1 mRNA were calculated based on PCR efficiency and the threshold cycle difference for the test sample (PBMCs from periodontitis patients) versus a calibrator (PBMCs from one healthy volunteer). Target gene expression was normalized using GAPDH gene expression (Pfaffl, 2001). The mRNA level of the calibrator was set to 1 .

\subsection{IL-1 $\beta$ production assay}

PBMCs were plated at a density of $2.0 \times 10^{5}$ cells per well in a 96well dish in triplicate and stimulated with $500 \mu \mathrm{g} / \mathrm{mL}$ silica crystals or PBS. Following incubation for $4 \mathrm{~h}$, the cell-free supernatant was harvested and analyzed for IL-1 $\beta$ secretion using an enzyme-linked immunosorbent assay (ELISA) kit (R\&D, Minneapolis, MN) according to the manufacture's protocol.

\subsection{Quantification of bacteria}

The levels of a species of periodontopathic bacterium, Porphyromonas gingivalis, and total bacteria in each saliva sample were determined as described by Yoshioka, Yoshimura, Kaneko, Golenbock, and Hara (2008), with slight modifications. Briefly, bacterial DNA was extracted from $20 \mu \mathrm{L}$ of the saliva samples, using the DNeasy tissue kit (Qiagen), and quantified using the Mx3000 P qPCR System. The sequences of the specific primers and probes used for each bacterium and 

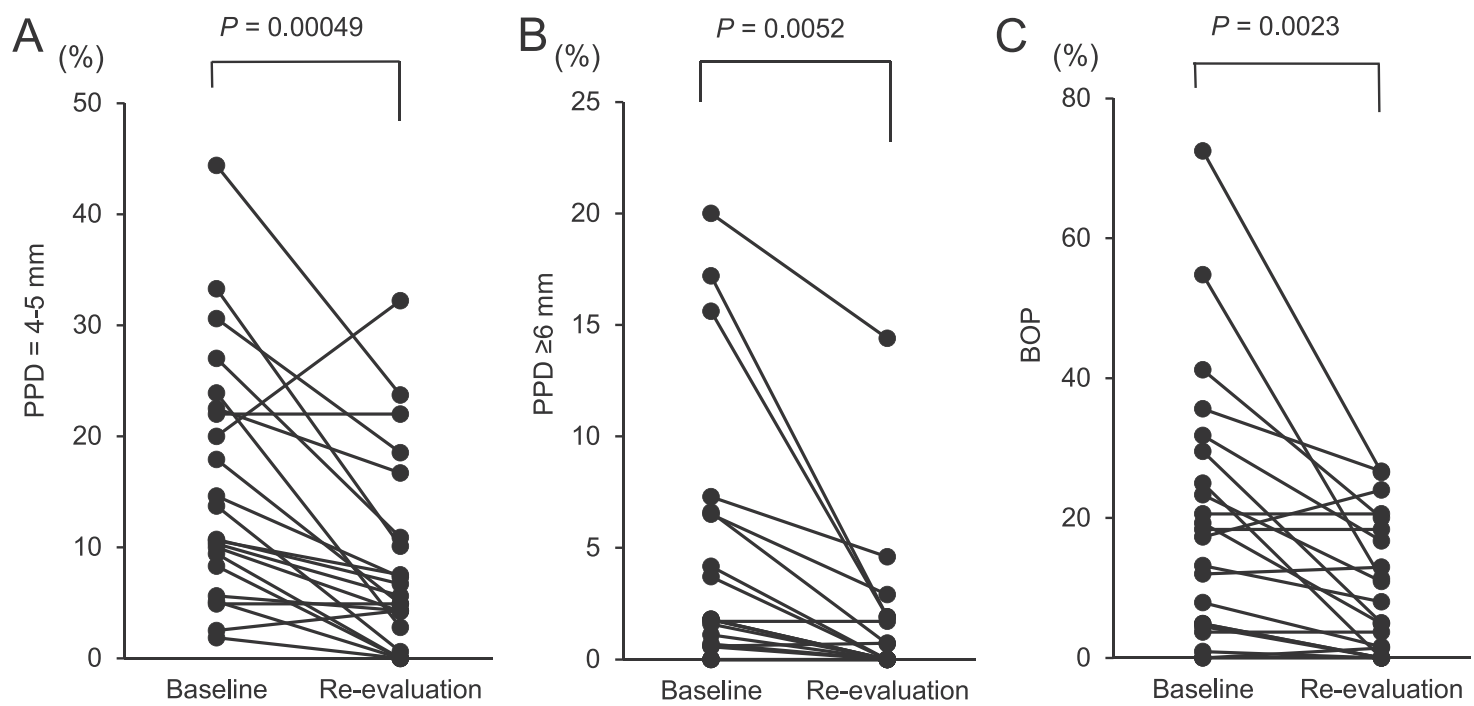

Fig. 1. Periodontal condition of the patients at baseline and re-evaluation.

Percentage of sites with PPD $=4-5 \mathrm{~mm}(\mathrm{~A})$, PPD $\geq 6 \mathrm{~mm}$ (B), or BOP (C), for each patient measured at baseline and re-evaluation.
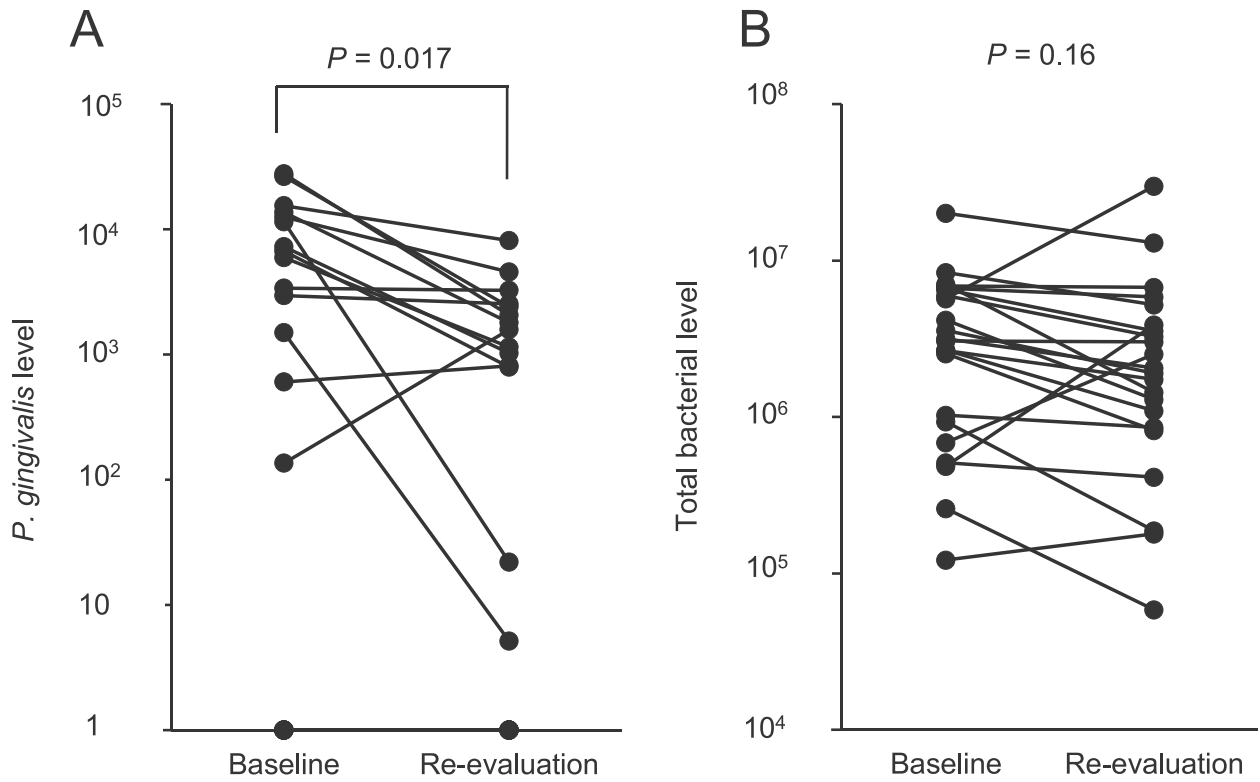

Fig. 2. Bacterial levels in saliva samples collected at baseline and re-evaluation.

Saliva samples were collected from each patient before and after treatment. The levels of $P$. gingivalis (A) and total bacteria (B) in $1 \mu \mathrm{L}$ of each saliva sample were determined using real-time PCR.

for total bacteria have been described elsewhere (Maeda et al., 2003; Pan et al., 2017). Standard curves for each organism were plotted for each primer set using threshold cycle values obtained by amplifying 10fold serial dilutions from 1 to $10^{7} \mathrm{CFU}$ of previously quantified bacterial DNA. Bacterial DNA standards and sample DNA were run in triplicate, and their means were used to calculate the bacterial concentrations.

\subsection{Statistical analysis}

Comparisons of the values at baseline and re-evaluation were performed using paired-sample $t$-tests. All calculations were conducted using StatMate IV software (ATMS, Tokyo, Japan).

\section{Results}

\subsection{Clinical parameters and bacterial levels of the saliva samples}

The mean percentage of sites with PPD $=4-5 \mathrm{~mm}$ was $15.9 \%$ before treatment and $8.5 \%$ after treatment (Fig. 1A). The mean percentage of sites with PPD $\geq 6 \mathrm{~mm}$ decreased from $4.3 \%$ to $1.4 \%$ (Fig. 1B), and that of BOP decreased from $20.1 \%$ to $11.8 \%$ (Fig. 1C). The mean levels of $P$. gingivalis in the saliva decreased from 220 to 78 after treatment (Fig. 2A), whereas it was not detected in the saliva of three healthy subjects (data not shown). The level of total bacteria did not change (Fig. 2B). These results indicate the effectiveness of the initial periodontal treatment. 
A

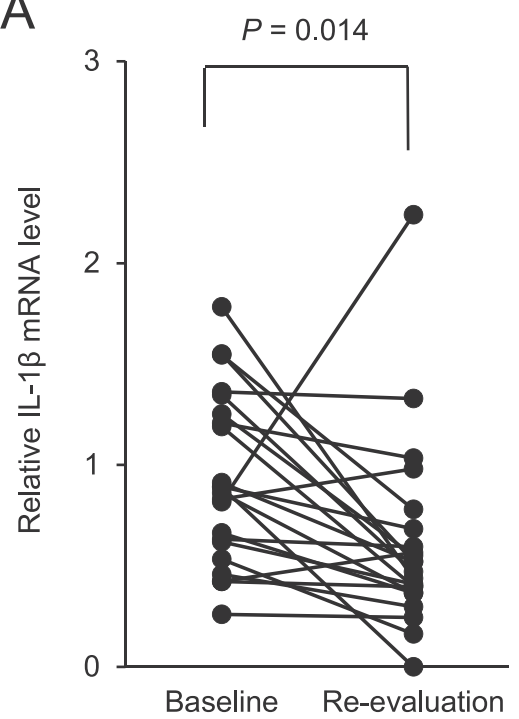

C

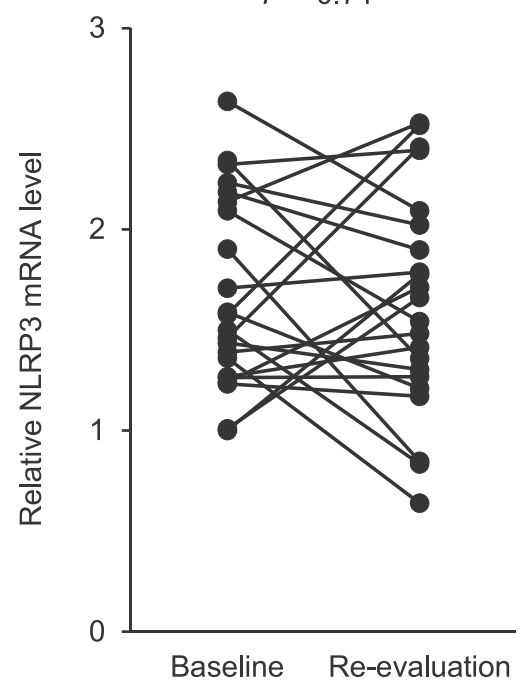

B

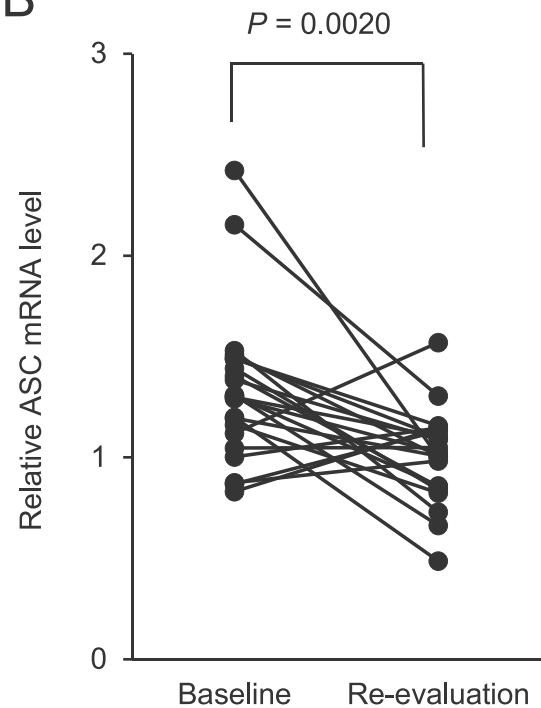

D

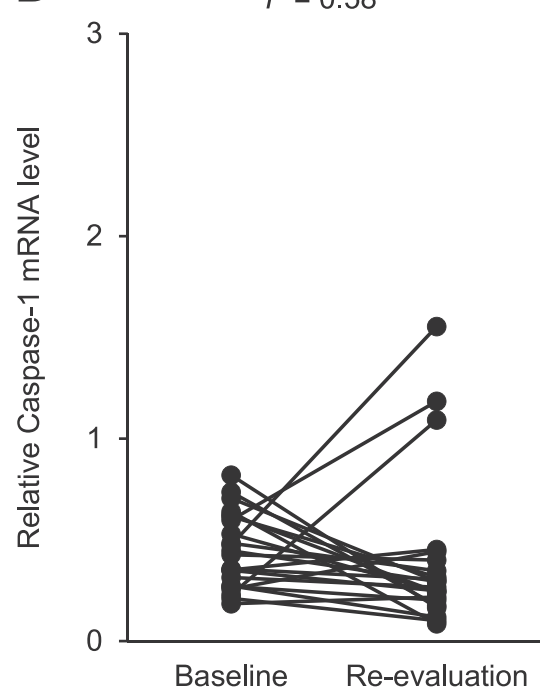

Fig. 3. Expression of $I L-1 \beta, A S C, N L R P 3$ and Caspase-1 mRNA in the PBMCs before and after periodontal treatment.

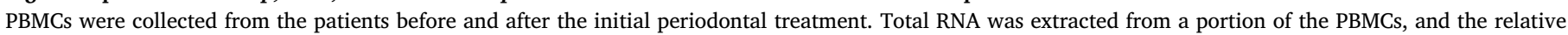
expression of $I L-1 \beta$ (A), ASC (B), NLRP3 (C), and Caspase-1 (D) mRNA was determined using qRT-PCR.

\subsection{Expression levels of IL-1B, NLRP3, ASC, and Caspase-1 mRNA in PBMCs}

PBMCs were collected from the patients before and after the treatment, and their expression levels of $I L-1 \beta, N L R P 3, A S C$, and Caspase-1 mRNA were analyzed using qRT-PCR. The mRNA levels of $I L-1 \beta$ and $A S C$ were significantly lower after the initial periodontal treatment (Fig. 3A and B). However, the mRNA levels of NLRP3 and Caspase-1 did not change (Fig. 3C and D). To further analyze the effect of periodontal treatment on inflammasome priming, the patients were classified according to their BOP percentage. Patients with BOP $\geq 16 \%$ are known to have a higher periodontal disease activity (Lang, Joss, Orsanic, Gusberti, \& Siegrist, 1986). The levels of $I L-1 \beta$ mRNA in the PBMCs decreased except two samples and the levels of ASC and NLRP3 mRNA significantly decreased after treatment in the BOP $<16 \%(n=10)$ group (Fig. 4A-C). The levels of Caspase-1 mRNA in the PBMCs did not change in the same group (Fig. 4D). The levels of $I L-1 \beta$, ASC and Caspase- 1 mRNA in the PBMCs also decreased after treatment in the BOP $\geq$ $16 \%(\mathrm{n}=12)$ group (Fig. 4E-H). However, the level of NLRP3 mRNA in the PBMCs slightly increased after treatment in the BOP $\geq 16 \%$ group (Fig. 4G).

\subsection{IL-1 $\beta$ secretion by PBMCs stimulated with silica crystals}

The PBMCs were then stimulated with PBS or silica crystals. Silica crystals were used as stimulant because they engage the NLRP3 inflammasome without priming the cells (Dostert et al., 2008). PBMCs stimulated with PBS did not secrete any IL- $1 \beta$, whereas those stimulated with silica crystals did (Fig. 5A and B). However, the levels of IL-1 $\beta$ thus induced did not change significantly during the treatment in the whole samples. Similarly to NLRP3 mRNA levels, the induction of IL-1 $\beta$ by silica crystals slightly decreased after treatment in the BOP $<16 \%$ group but increased in the $\mathrm{BOP} \geq 16 \%$ group (Fig. $5 \mathrm{C}$ and D).

\section{Discussion}

In this study, we analyzed the effects of initial periodontal treatment on inflammasome priming in PBMCs. Periodontal treatment reduced the percentage of sites with deep PPD and/or BOP in every patient. The levels of $P$. gingivalis in the saliva samples decreased after the treatment, 
A

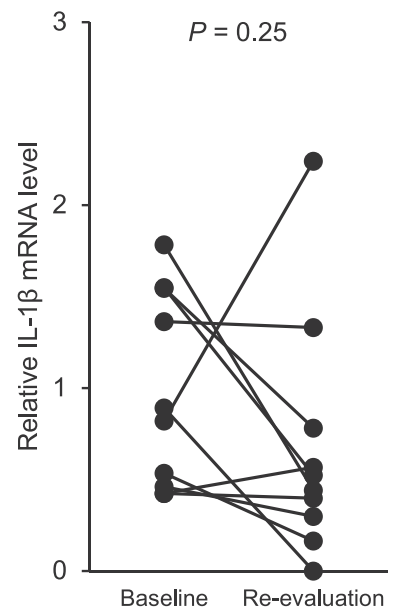

B

$E$

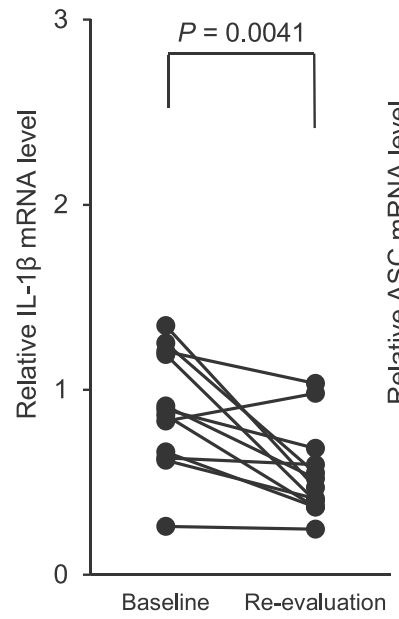

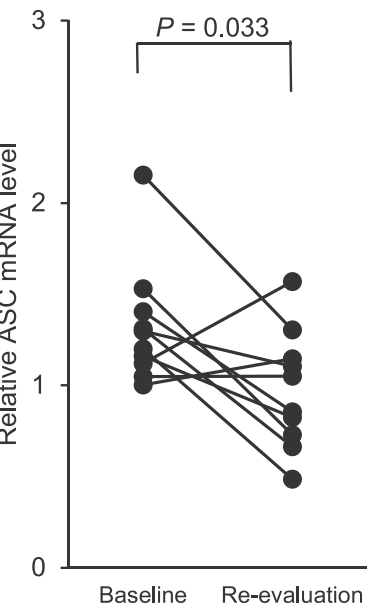

$\mathrm{F}$

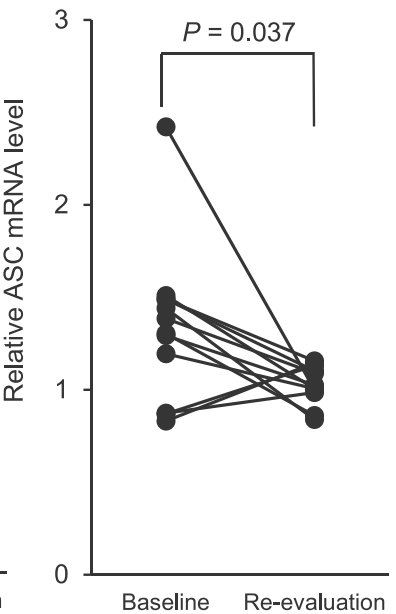

C

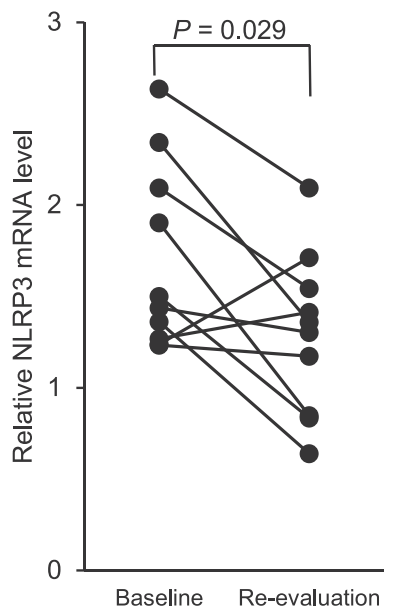

G

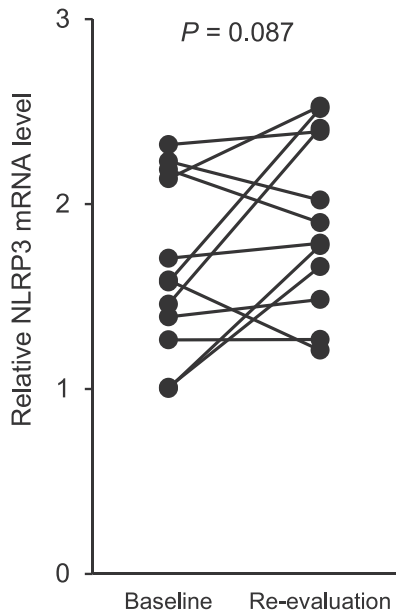

D

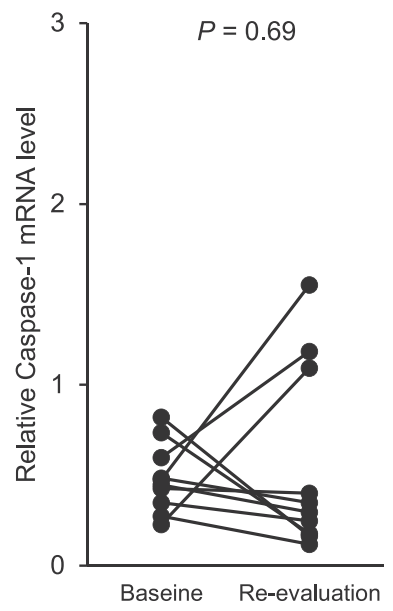

$\mathrm{H}$

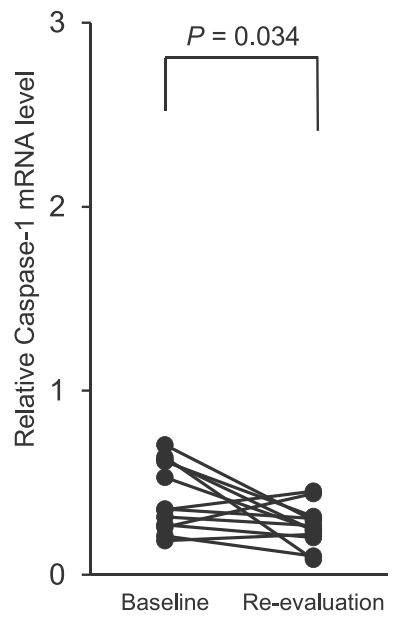

Fig. 4. Expression of $I L-1 \beta$, ASC, NLRP3, and Caspase-1mRNA in the PBMCs by BOP percentages.

The patients were classified into two groups according to their BOP percentages. The relative expression of IL-1 $\beta$ (A, E), ASC (B, F), NLRP3 (C, G), and Caspase-1 (D, $\mathrm{H})$ mRNA levels in the BOP $<16 \%$ group (A-D) and BOP $\geq 16 \%$ group (E-H) were shown.

confirming the effectiveness of the treatment (Saygun et al., 2011). Concurrently, mRNA expression levels of $I L-1 \beta$ and ASC in the PBMCs decreased after the treatment, suggesting that it contributed to the downregulation of $I L-1 \beta$ and ASC mRNA in the PBMCs. There are many factors that can activate the inflammasome, such as ATP from dead cells, metal powder, and ultraviolet B (Eisenbarth, Colegio, O'Connor, Sutterwala, \& Flavell, 2008; Feldmeyer et al., 2007; Mariathasan et al., 2006). The expression levels of $I L-1 \beta, N L R P 3$, ASC and Caspase-1 mRNA varied widely, indicating varying levels of inflammasome priming. Nevertheless, $I L-1 \beta$ and ASC mRNA levels decreased after the periodontal treatment, suggesting the substantial contribution of periodontal diseases to the inflammasome priming of PBMCs.

However, NLRP3 and Caspase-1 mRNA did not decrease after treatment. Unassociated regulation of NLRP3 and ASC expression was found in the previous studies by other investigators. NLRP3 expression was enhanced but ASC expression was downregulated in Mono-Mac-6 cells stimulated with $P$. gingivalis (Bostanci et al., 2009). NLRP3 expression level was elevated in periodontally diseased groups but the expression level of ASC was similar between groups (Aral et al., 2019). To explore the reason for the unchanged NLRP3 mRNA levels, the patients were classified into two groups based on their BOP percentage. $\mathrm{BOP}$ reflects the histological, clinical, and bacteriological alterations associated with periodontal diseases (Greenstein, 1984). ASC and
NLRP3 mRNA expression of patients with BOP $<16 \%$ significantly decreased, whereas $I L-1 \beta$ and caspase- 1 mRNA expression did not. $I L-1 \beta$ and caspase- 1 mRNA levels ( 0.90 and 0.48 , respectively) at baseline were lower than ASC and NLRP3 mRNA levels (1.32 and 1.70, respectively). Less up-regulation of $I L-1 \beta$ and caspase-1 mRNA at baseline might result in non-significant changes after the treatment. NLRP3 mRNA expression levels of patients with BOP $\geq 16 \%$ slightly increased, whereas those of patients with BOP $<16 \%$ slightly decreased after treatment. Because the BOP percentage of the former group remained high, the treatment was not able to completely eliminate the inflammation in their periodontal tissue, and the residual inflammation might have contributed to the slightly increased NLRP3 mRNA expression in their PBMCs. Alternatively, it has been shown that subgingival biofilms containing $P$. gingivalis down-regulates NLRP3, but not ASC and caspase-1 expression (Belibasakis, Guggenheim, \& Bostanci, 2013). As shown in Fig. 2, the level of $P$. gingivalis decreased after the treatment. The reduction of the level of $P$. gingivalis might attenuate the inhibitory effect and contribute to the up-regulation of NLRP3 of patients with $\mathrm{BOP} \geq 16 \%$. In addition, chronic inflammation in the periodontal tissue might cause innate immune cells to be reprogrammed to resist to the reduction of NLRP3 mRNA and IL- $1 \beta$ protein levels. It has been shown that long-term epigenetic reprogramming of innate immune cells, also termed "trained immunity," causes prolonged 
A

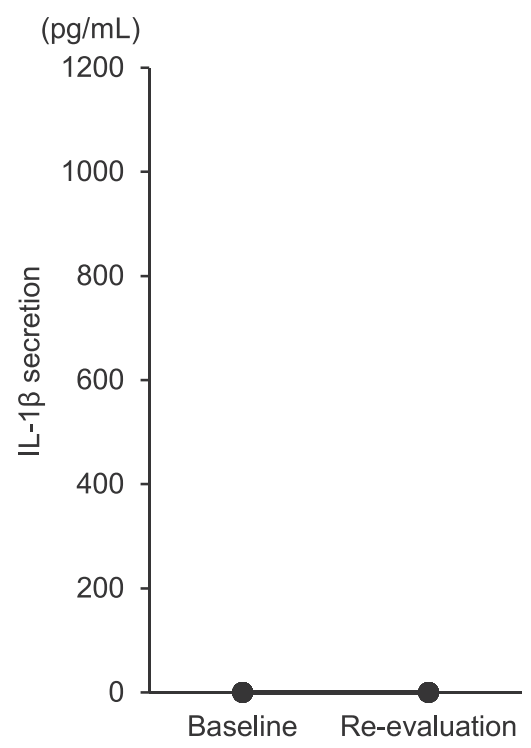

C

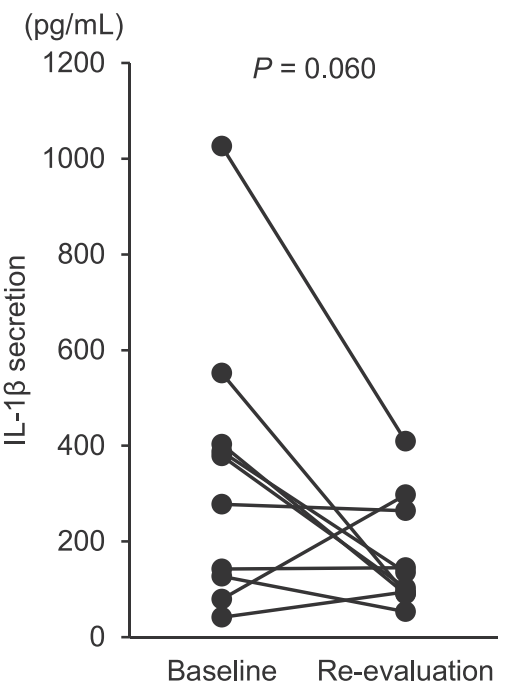

B

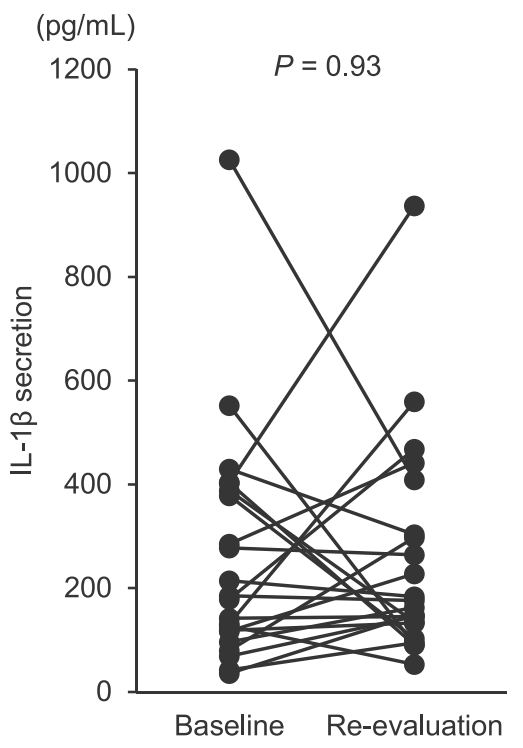

$\mathrm{D}$

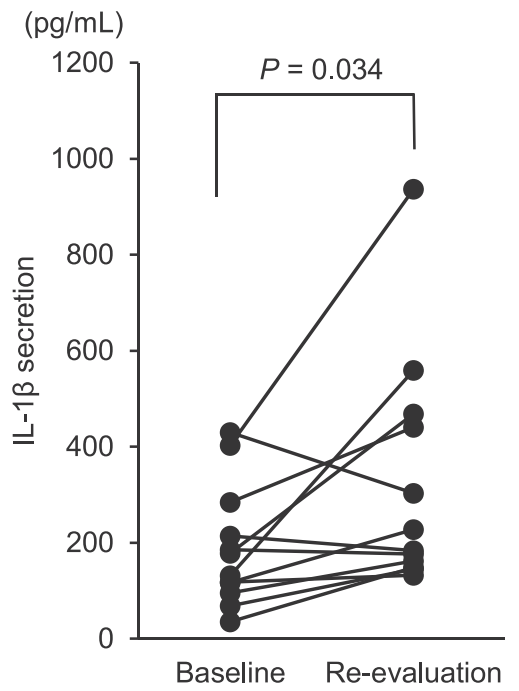

Fig. 5. IL-1 $\beta$ secretion levels before and after treatment.

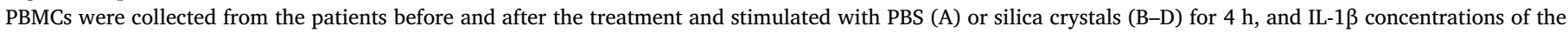

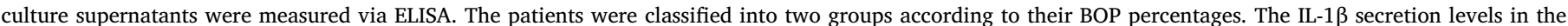
$\mathrm{BOP}<16 \%$ group (C) and BOP $\geq 16 \%$ group (D) were shown.

altered cellular functionality for more than 4 weeks (Christ et al., 2018). The PBMCs in this study were collected $41.1 \pm 29.1 \mathrm{~d}$ after treatment, which may not have been long enough to resolve the inflammasome priming. The long-term effects of periodontal treatment on inflammasome priming should therefore be investigated in future studies with a longer observational period.

PBMCs stimulated with silica crystals secreted IL- $1 \beta$, whereas those stimulated with PBS did not. Because silica crystals do not prime the cells (Dostert et al., 2008), these results suggest that the PBMCs had already been primed prior to blood sampling. The secretion of IL- $1 \beta$ by silica crystals slightly decreased after treatment in the BOP $<16 \%$ group but increased in the BOP $\geq 16 \%$ group. The decrease in the levels of IL- $1 \beta$ secretion in the BOP $<16 \%$ group might have been due to the reduction of ASC and NLRP3 mRNA expression. The increase in the levels of IL- $1 \beta$ secretion in the BOP $\geq 16 \%$ group might have been due to the increase of NLRP 3 mRNA expression. Periodontal pathogens can trigger IL-1 $\beta$ production and upregulate the IL-1 $\beta$ concentrations of the gingival crevicular fluids and serum of patients with periodontitis
(Hegde \& Awan, 2018; Zhu, Lin, Zheng, \& Chen, 2015). In addition, bacteria harbored in the periodontal tissue can gain access to the circulatory system, resulting in a state of transient bacteremia. These bacteria or bacterial products can stimulate the infiltrated macrophages to induce IL-1 $\beta$ production in the lesions of systemic diseases (Falcao \& Bullón, 2019). However, circulating IL-1 $\beta$ or bacteremia can affect not only the disease lesions but also any tissue in the body. In contrast to the circulating IL-1 $\beta$ or bacteria, primed PBMCs would not induce inflammation other than the target organs. DAMPs such as monosodium urate, cholesterol, and $\beta$-amyloid crystals activate the NLRP3 inflammasome in the lesions of gout, atherosclerosis, and Alzheimer's disease. Without this stimulation by DAMPs, primed PBMCs would not secrete IL-1 $\beta$, so their effects would be limited to the target organs. We have demonstrated that the inflammasome priming status of PBMCs were altered by periodontal treatment. The results of the present study are the first evidence suggesting that inflammation in the periodontal tissue can prime PBMCs, which may affect the symptoms of systemic diseases. 
In conclusion, the initial periodontal treatment improved periodontal conditions and altered the inflammasome priming status of the PBMCs, in which $I L-1 \beta, A S C, N L R P 3$ and Caspase-1 mRNA expression was differentially regulated in patients with $\mathrm{BOP}<16 \%$ and $\mathrm{BOP} \geq$ $16 \%$. The distinct inflammasome priming status by BOP percentages could be due to the residual inflammation in periodontal tissue or the effects of $P$. gingivalis in dental plaque. It is necessary to use a long observational period to confirm the effects of complete periodontal treatment, including periodontal surgery, on inflammasome priming.

\section{Declaration of Competing Interest}

The authors declare no potential conflicts of interest with respect to the authorship and/or publication of this article.

\section{Acknowledgements}

This work was supported by a Grant-in-Aid for Scientific Research (C)16K11836 from the Japan Society for the Promotion of Science, Tokyo, Japan.

\section{References}

Aral, K., Berdeli, E., Cooper, P. R., Milward, M. R., Kapila, Y., Karadede Ünal, B., ... Berdeli, A. (2019). Differential expression of inflammasome regulatory transcripts in periodontal disease. Journal of Periodontology, 1-11. https://doi.org/10.1002/JPER. 19-0222.

Belibasakis, G. N., Guggenheim, B., \& Bostanci, N. (2013). Down-regulation of NLRP3 inflammasome in gingival fibroblasts by subgingival biofilms: Involvement of Porphyromonas gingivalis. Innate Immunity, 19(1), 3-9.

Bostanci, N., Emingil, G., Saygan, B., Turkoglu, O., Atilla, G., Curtis, M. A., ... Belibasakis, G. N. (2009). Expression and regulation of the NALP3 inflammasome complex in periodontal diseases. Clinical and Experimental Immunology, 157(3), 415-422.

Christ, A., Günther, P., Lauterbach, M. A. R., Duewell, P., Biswas, D., Pelka, K., ... Latz, E. (2018). Western diet triggers NLRP3-dependent innate immune reprogramming. Cell, 172(1-2) 162-175.e114.

Dostert, C., Pétrilli, V., Van Bruggen, R., Steele, C., Mossman, B. T., \& Tschopp, J. (2008). Innate immune activation through Nalp3 inflammasome sensing of asbestos and silica. Science, 320(5876), 674-677.

Duewell, P., Kono, H., Rayner, K. J., Sirois, C. M., Vladimer, G., Bauernfeind, F. G., \& Latz, E. (2010). NLRP3 inflammasomes are required for atherogenesis and activated by cholesterol crystals that form early in disease. Nature, 464(7293), 1357-1361.

Eisenbarth, S. C., Colegio, O. R., O'Connor, W., Sutterwala, F. S., \& Flavell, R. A. (2008). Crucial role for the Nalp3 inflammasome in the immunostimulatory properties of aluminium adjuvants. Nature, 453(7198), 1122-1126.

Emsley, H. C., Smith, C. J., Georgiou, R. F., Vail, A., Hopkins, S. J., Rothwell, N. J., ... Acute Stroke Investigators (2005). A randomised phase II study of interleukin-1 receptor antagonist in acute stroke patients. Journal of Neurology, Neurosurgery, and
Psychiatry, 76(10), 1366-1372.

Falcao, A., \& Bullón, P. (2019). A review of the influence of periodontal treatment in systemic diseases. Periodontology 2000, 79(1), 117-128.

Feldmeyer, L., Keller, M., Niklaus, G., Hohl, D., Werner, S., \& Beer, H. D. (2007). The inflammasome mediates UVB-induced activation and secretion of interleukin- $1 \beta$ by keratinocytes. Current Biology, 17(13), 1140-1145.

Feria, M. G., Taborda, N. A., Hernandez, J. C., \& Rugeles, M. T. (2018). HIV replication is associated to inflammasomes activation, IL-1 $\beta$, IL-18 and caspase-1 expression in GALT and peripheral blood. PLoS One, 13(4), e0192845.

Gaur, S., \& Agnihotri, R. (2015). Alzheimer's disease and chronic periodontitis: Is there an association? Geriatrics \& Gerontology International, 15(4), 391-404.

Greenstein, G. (1984). The role of bleeding upon probing in the diagnosis of periodontal disease. A literature review. Journal of Periodontology, 55(12), 684-688.

He, Y., Hara, H., \& Núñez, G. (2016). Mechanism and regulation of NLRP3 inflammasome activation. Trends in Biochemical Sciences, 41(12), 1012-1021.

Hegde, R., \& Awan, K. H. (2018). Effects of periodontal disease on systemic health. Disease-A-Month, 65(6), 185-192.

Ishida, N., Ishihara, Y., Ishida, K., Tada, H., Funaki-Kato, Y., Hagiwara, M., ... Matsushita, K. (2017). Periodontitis induced by bacterial infection exacerbates features of Alzheimer's disease in transgenic mice. NPJ Aging and Mechanisms of Disease, 3, 15.

Lang, N. P., Joss, A., Orsanic, T., Gusberti, F. A., \& Siegrist, B. E. (1986). Bleeding on probing. A predictor for the progression of periodontal disease? Journal of Clinical Periodontology, 13(6), 590-596.

Lukens, J. R., Gross, J. M., \& Kanneganti, T. D. (2012). IL-1 family cytokines trigger sterile inflammatory disease. Frontiers in Immunology, 3, 315.

Maeda, H., Fujimoto, C., Haruki, Y., Maeda, T., Kokeguchi, S., Petelin, M., ... Takashiba, S. (2003). Quantitative real-time PCR using TaqMan and SYBR Green for Actinobacillus actinomycetemcomitans, Porphyromonas gingivalis, Prevotella intermedia, tetQ gene and total bacteria. FEMS Immunology and Medical Microbiology, 39(1), 81-86.

Mariathasan, S., Weiss, D. S., Newton, K., McBride, J., O’Rourke, K., Roose-Girma, M., ... Dixit, V. M. (2006). Cryopyrin activates the inflammasome in response to toxins and ATP. Nature, 440(7081), 228-232.

Pan, S., Liu, Y., Zhang, L., Li, S., Zhang, Y., Liu, J., ... Xiao, S. (2017). Profiling of subgingival plaque biofilm microbiota in adolescents after completion of orthodontic therapy. PLoS One, 12(2), e0171550.

Patel, M. N., Carroll, R. G., Galván-Peña, S., Mills, E. L., Olden, R., Triantafilou, M., ... Masters, S. L. (2017). Inflammasome priming in sterile inflammatory disease. Trends in Molecular Medicine, 23(2), 165-180.

Pfaffl, M. W. (2001). A new mathematical model for relative quantification in real-time RT-PCR. Nucleic Acids Research, 29(9), e45.

Saygun, I., Nizam, N., Keskiner, I., Bal, V., Kubar, A., Açıkel, C., ... Slots, J. (2011) Salivary infectious agents and periodontal disease status. Journal of Periodontal Research Supplement, 46(2), 235-239.

Teeuw, W. J., Slot, D. E., Susanto, H., Gerdes, V. E., Abbas, F., D'Aiuto, F., ... Loos, B. G. (2014). Treatment of periodontitis improves the atherosclerotic profile: A systematic review and meta-analysis. Journal of Clinical Periodontology, 41(1), 70-79.

Tzach-Nahman, R., Nashef, R., Fleissig, O., Palmon, A., Shapira, L., Wilensky, A., \& Nussbaum, G. (2017). Oral fibroblasts modulate the macrophage response to bacterial challenge. Scientific Reports, 7(1), 11516.

Yoshioka, H., Yoshimura, A., Kaneko, T., Golenbock, D. T., \& Hara, Y. (2008). Analysis of the activity to induce Toll-like receptor (TLR)2- and TLR4-mediated stimulation of supragingival plaque. Journal of Periodontology, 79(5), 920-928.

Zhu, H., Lin, X., Zheng, P., \& Chen, H. (2015). Inflammatory cytokine levels in patients with periodontitis and/or coronary heart disease. International Journal of Clinical and Experimental Pathology, 8(2), 2214-2220. 